\title{
MEASUREMENTS ON THE FIRST LHC ACCELERATION MODULE
}

\author{
E. Chiaveri, O. Brunner, E. Ciapala, H. Frischholz, H.P. Kindermann, T. Linnecar, R. Losito, \\ E. Montesinos and J. Tückmantel, CERN, Geneva, Switzerland
}

\section{Abstract}

The Large Hadron Collider (LHC), now under construction at CERN, will accelerate the two counterrotating beams of protons from an injection energy of $450 \mathrm{GeV}$ to the coast value of $7 \mathrm{TeV}$ where they will collide. The main accelerating system for each beam comprises eight $400 \mathrm{MHz}$ superconducting cavities giving a total of $16 \mathrm{MV}$. The cavities are grouped in fours in cryogenic modules to ease installation and reduce heat losses. The different requirements at injection and collision together with the heavy beam loading have imposed the use of a variable power coupler (the coupling can be varied by a factor 10) and a tuner with $180 \mathrm{kHz}$ range. The $300 \mathrm{~kW}$ power is supplied by a klystron attached to the cavity via a circulator. The first module with its four cavities has now been assembled at CERN and tested. The results from these measurements are presented, problems encountered are described and modifications to be applied in the future are given.

\section{INTRODUCTION}

In the LHC each beam will be accelerated by eight superconducting single-cell cavities resonating at $400.8 \mathrm{MHz}$ [1]. The cavities are grouped in fours in one single cryostat $8 \mathrm{~m}$ long to reduce cost and cryogenic losses (Fig. 1).

The main components of the LHC module are:
- the cavities, with their stainless steel helium vessel, made with the same technology successfully used in the CERN LEP collider (copper cavities with a film of niobium sputtered on the RF surface)

- the mechanical tuner of $180 \mathrm{kHz}$ stroke, that ensures high speed with a resolution of $\sim 20 \mathrm{~Hz}$

- the insulation vacuum vessel, built in one piece with no welds

- the HOM couplers, four per cavity, two for longitudinal modes and two for transverse modes

- the main coupler, a complex mechanical construction capable of changing the coupling to the cavity by a factor of at least 10 , to cope with the different modes of operation in the LHC (injection, ramping, storage)

- the second beam pipe: in the LHC the two counterrotating beams will run in two separate pipes and only one beam will pass through each cavity. The other beam will travel through a pipe installed inside the cryostat (but outside the cavity) at a distance of $420 \mathrm{~mm}$ from the cavity axis (Fig. 2).

We decided to produce 21 cavities in order to have a complete spare module plus a spare cavity for quick replacement of faulty cavities in case of problems during operation. All 21 cavities have already been tested separately in vertical cryostats and all reached an average accelerating field of $9 \mathrm{MV} / \mathrm{m}$ with a Q-factor bigger than $\left.10^{9}[2]\right)$. Only three of them had to be coated a second

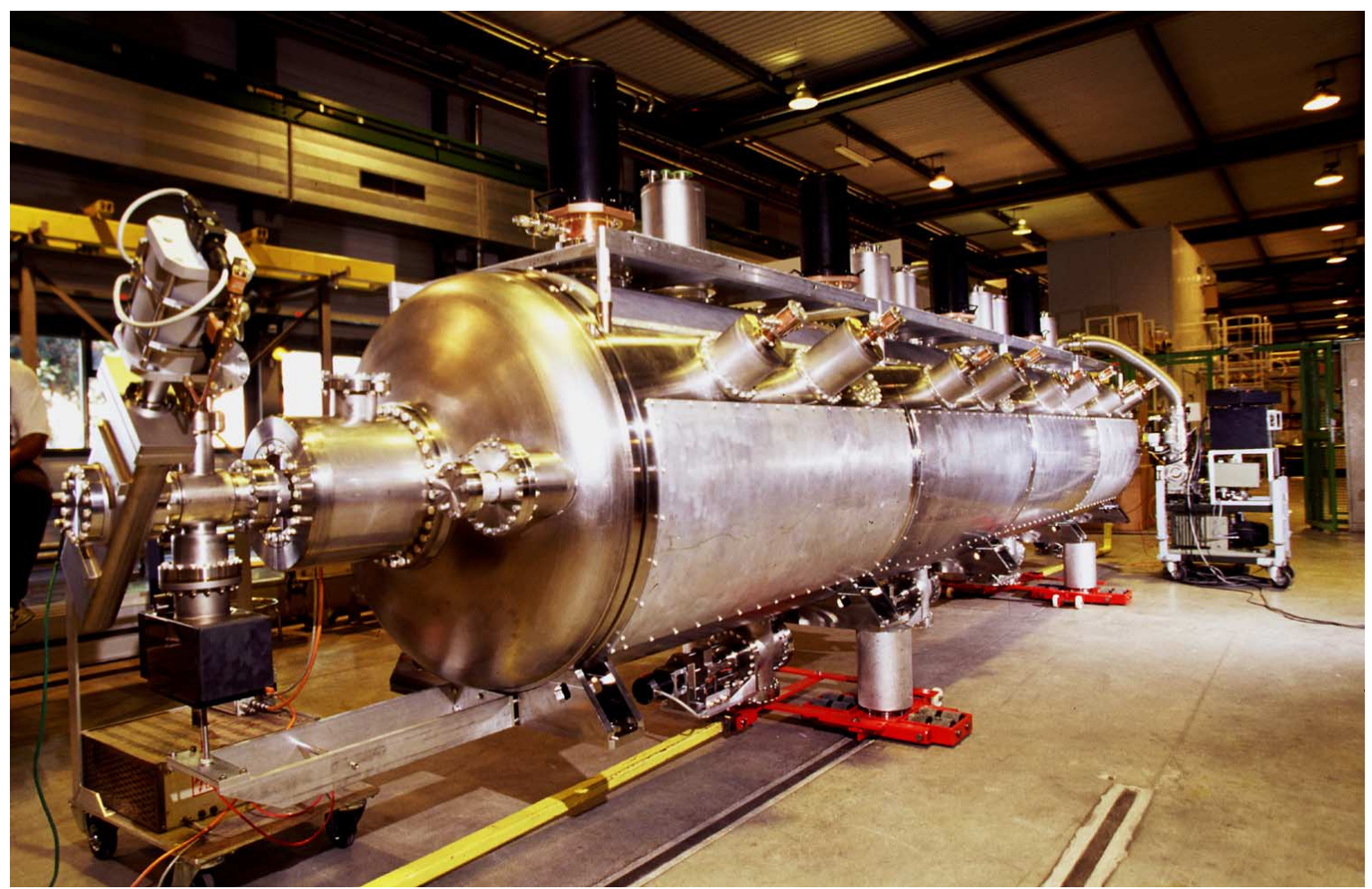

Figure 1 One complete RF module 


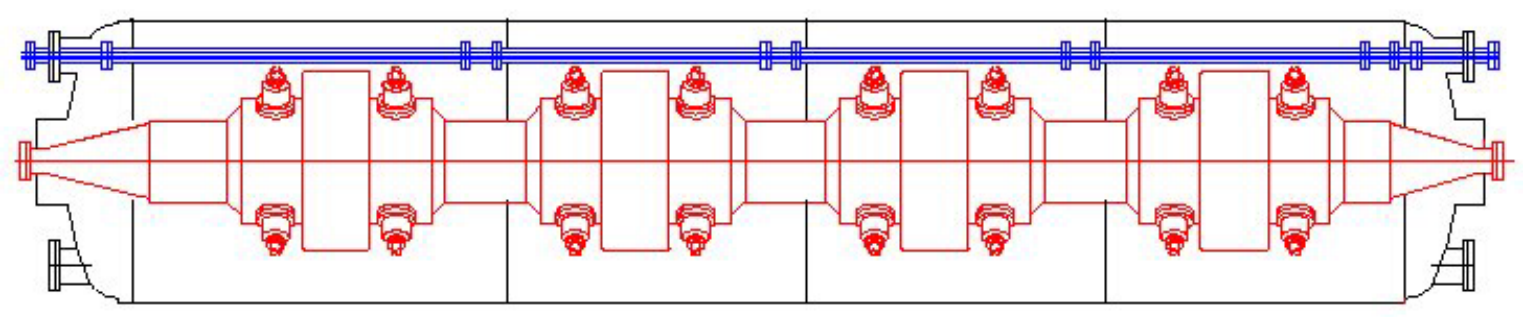

Figure 2 The second beam pipe installed in the RF module cryostat

time to achieve this performance.

All the cavities have been equipped with their helium vessel, ready for installation in the modules. Three modules have already been assembled at CERN and the others will be ready for low-level measurements by the end of the year. Each module can be assembled in six weeks, including clean-room work (such as interconnection of the four cavities and installation of HOM and power couplers) and all other activities (welding of He pipes, assembly of the mechanical tuner, installation of thermometers and superinsulation, HOM couplers tuning and installation of the insulation vessel covers).

In November 2000 the first module of the LHC $400 \mathrm{MHz}$ superconducting system was tested at $4.5 \mathrm{~K}$ up to $300 \mathrm{KW}$ per cavity at the nominal field $(5.3 \mathrm{MV} / \mathrm{m})$. The goal of this test was to validate the overall system design with four cavities in one cryostat at cryogenic temperature and full RF power. Several details were to be measured for the first time, e.g. the cryogenic static losses, the tuning range of the tuner in its final configuration and eventual crosstalk on the fundamental mode between the cavities. For some other parameters we had to confirm the values previously measured on the two-cavity prototype module:

- the possibility to go up to $9 \mathrm{MV} / \mathrm{m}$ (even if the nominal field is $5 \mathrm{MV} / \mathrm{m}$ )

- the ability of the power coupler to work safely up to $300 \mathrm{~kW}$ with full reflection

- the rejection of the fundamental mode by the High Order Modes (HOM) couplers

- the sensitivity of the cavity to pressure variations

The tests were performed in the installations housed in building SM18 at CERN, where the cold test of LHC superconducting magnets will also be done. The cryogenic plant consists of one refrigerator capable of absorbing6 kW@4.5 K with full recovery of the helium consumed.

In the bunker in SM18 (see Fig. 1) we can perform both low power (with $200 \mathrm{~W}$ solid state amplifiers) and high power tests. A $1 \mathrm{MW}$ klystron, basically a LEP $352 \mathrm{MHz}$ klystron modified to work at $400 \mathrm{MHz}$, is installed next to the bunker and can feed the four cavities simultaneously via a circulator and a network of "magic Tees". The results of the measurements are presented in the next section.

\section{THE RESULTS}

\subsection{The Cooldown}

During the first cooldown the cavity cooled down very quickly (in one hour it was already below $70 \mathrm{~K}$ ) but its flanges, connected to the massive stainless steel plates of the tuner mechanism (more than $300 \mathrm{~kg}$ ) needed much more time to arrive at thermal equilibrium (about $48 \mathrm{~h}$ ). Under these conditions much of the cooling power of the cold He gas coming in was not used. For this reason we limited the aperture of the input valve in the following cooldowns to improve the efficiency of the process and to reduce all the mechanical stresses due to non-negligible temperature gradients. At present the cavities take 7 to $8 \mathrm{~h}$ to fill with helium, but the thermal stability is reached for the whole system after only $\sim 48 \mathrm{~h}$.

\subsection{HOM Coupler}

A good setting for the HOM couplers was found after a few attempts. The HOM couplers are of two different types [3] and are pre-tuned at $300 \mathrm{~K}$ in a dummy cavity by plastic deformation of the antenna to obtain the correct orientation in order to reject the fundamental mode $(399.010 \pm 0.030 \mathrm{MHz} @ 300 \mathrm{~K})$ by more than $100 \mathrm{~dB}$. After that, they are chemically polished, rinsed in ultraclean water and then mounted on the cavities in a clean room. At this stage the couplers for dipolar modes (type "A") are very rigid and can no longer be deformed, so their final tuning is done by turning the antenna until the rejection of the fundamental mode at $300 \mathrm{~K}$ is again better than $100 \mathrm{~dB}$ with respect to RF power injected on the pick-up antenna on the opposite side of the cavity $\left(\mathrm{Q}_{\text {ext }} \sim 10^{11}\right)$. For longitudinal mode couplers (type "B") it has been found by measurements that a rejection of less than $80 \mathrm{~dB}$ of the fundamental mode at $300 \mathrm{~K}$ gives better rejection after cooldown. Type "B" couplers can be elastically deformed to obtain the correct rejection value using a clamp mounted on it.

At $4.5 \mathrm{~K}$ the measured external $\mathrm{Q}$ was above $2 \cdot 10^{10}$ for type "A" couplers, and higher than $10^{11}$ for type "B". (It was not possible to detect any power coming out on the fundamental mode at nominal field.) We tested their cooling efficiency under heavy RF power by forcing the transmission of $500 \mathrm{~W}$ at $600 \mathrm{MHz}$ between two couplers and we did not observe any quench. 
The damping of HOM was measured only on the twocavity prototype. For all the modes that we could measure, the loaded Q was well within the specifications. More detailed measurement will be done in future.

\subsection{Cavity Conditioning}

The cavities did not require any special conditioning to reach the nominal field. During low-power tests, both in the vertical cryostat and in the final horizontal cryostat they showed a multipacting band between 5 and $7 \mathrm{MV} / \mathrm{m}$, that could easily be conditioned by the combined action of $\mathrm{He}$ processing and RF processing. After low-level measurements the critically coupled antenna is replaced in the clean room by the power coupler; we did not observe any deterioration of the cavity performance after this operation. We could easily reach the nominal field (within a few hours) and we had no evidence of multipacting at high power in the cavity. The maximum field achieved was $\sim 10 \mathrm{MV} / \mathrm{m}$, without any limitation.

\subsection{Power Coupler Conditioning.}

The LHC cavity power coupler [4] is more complex than the LEP one because the coupling to the cavity must be changed during operation by almost a factor 10 (from 16000 to 130000 ) for the different modes of operation. Input power levels up to $300 \mathrm{~kW}$ can be reached during operation and the power couplers must be conditioned in SM18 up to this value.

An automatic conditioning system combining direct control of the coupler vacuum (analogue feedback loop) with the computer control permitted increased efficiency. A first objective was to obtain maximum field in the cavities (weak coupling). The cavity conditioning was completed after about four days using approximately $45 \mathrm{~kW} \mathrm{CW}$ forward power on each coupler. After that, also in about four days, one of the couplers was also conditioned and tested to $200 \mathrm{~kW} \mathrm{CW}$ and $300 \mathrm{~kW}$ pulsed power (corresponding to equivalent peak power levels of $800 \mathrm{~kW}$ and $1200 \mathrm{~kW}$ respectively, due to the full reflection at the cavity input). It was also possible to condition this coupler at any phase, without instabilities, by simply detuning the cavity. This is an important result, since it permits complete conditioning of the LHC modules without beam.

\subsection{Tuner Performance}

The tuner is based on elastic mechanical deformation of the cavity [5]. The mechanism, specially designed to reduce backlash, is driven by a stepping motor with a maximum rate of $4000 \mathrm{steps} / \mathrm{s}$. The measured resolution in frequency is $20 \div 25 \mathrm{~Hz} / \mathrm{step}$, and its maximum speed is coherent with the above values: $\sim 100 \mathrm{kHz} / \mathrm{s}$. The tuning range is always greater than $180 \mathrm{kHz}$.

\subsection{Lorentz Detuning, Microphonics, Crosstalk.}

No problems of Lorentz detuning are foreseen since the cavities will be operated at constant field. Concerning microphonics, we measured a peak to peak deviation of $35 \mathrm{~Hz}$ from the nominal frequency in the worst case, given by a mechanical resonance of the cavity around $106 \mathrm{~Hz}$. This is not considered a problem since each cavity will be driven by its own klystron and strong RF feedback will be used, the bandwidth of the system being at least $2 \mathrm{kHz}$. The klystron has enough excess power to cope with this kind of problem. No influence of one cavity on the others was measured.

\subsection{Cryogenic losses.}

The measured static losses for the entire module are $150 \mathrm{~W}$. One third of these losses comes from the HOM couplers that are connected to the outside world through rigid lines. The heat flow is limited by an interruption of the external conductor on this line (RF continuity is insured by finger contacts) to $3 \mathrm{~W}$ for each coupler, bringing to nearly $50 \mathrm{~W}$ the contribution for the losses of the whole module by HOM couplers. The tapers from the cavity to the $300 \mathrm{~K}$ beam pipe on both ends of the module contribute $7 \mathrm{~W}$ each because they are copper-plated to reduce the impedance seen by the beam.

\section{CONCLUSIONS}

The first module of the LHC $400 \mathrm{MHz}$ superconducting system has been assembled and tested at CERN. No special problems were detected during this test and all the design parameters satisfy the requirements. By the end of 2001 all modules should be assembled and conditioning finished by the end of 2002 .

\section{ACKNOWLEDGEMENTS}

The authors wish to thank the many persons who contributed to the design and construction of the LHC SRF system.

\section{REFERENCES}

[1] Boussard, D., Linnecar, T., The LHC Superconducting RF System, Proc. 1999 Cryogenic Engineering Conference, Montreal, Canada, published in Advances in Cryogenic Engineering, Vol. 45, p. 385, 1999.

[2] Bauer, S. et al., Production of $\mathrm{Nb} / \mathrm{Cu}$ Sputtered Superconducting Cavities for LHC, Proc. 9th Workshop on RF Superconductivity, Santa Fe, New Mexico, USA, 1999.

[3] Haebel, E. et al., The Higher-order Mode Dampers of the $400 \mathrm{MHz}$ Superconducting LHC Cavities, Proc. 8th Workshop on RF Superconductivity, Abano Terme, Italy, 1997.

[4] Kindermann, H.-P., Stirbet, M., The Variable Power Coupler for the LHC Superconducting Cavity, Proc. 9th Workshop on RF Superconductivity, Santa Fe, New Mexico, USA, 1999.

[5] Boussard, D. et al., The LHC Superconducting Cavities, Proc. 1999 Particle Accelerator Conference, New York City, NY, USA,1999. 